\title{
3 \\ Acides gras mono-insaturés (oléique, palmitoléique et myristoléique) et leurs dérivés
}

> L'acide oléique se trouve-t-il uniquement dans l'huile d'olive?

> Notre organisme est-il capable de fabriquer de l'acide oléique?

> La consommation d'acide oléique à haute dose représente-t-elle un danger?

> Quelle différence entre les acides palmitique et palmitoléique?

> Où trouve-t-on de l'acide myristoléique?

> Le tissu adipeux contrôlerait-il notre poids ?

> L'acide érucique autrefois contenu dans le colza est-il dangereux?

> Quel rapport entre acides gras et inflammation ?

Dès les années 1955-1960, comme nous l'avons vu dans le premier chapitre consacré au besoin en lipides, J. Mead avait décrit la famille oléique en $n$-9 et la famille palmitoléique en $n-7$. Pourtant, pendant quelque 40 ans, à de rares exceptions près (pédiatres et néonatologues) 
peu de spécialistes des lipides s'intéressèrent à la famille palmitoléique. Le temps est venu de tenter de proposer une vision d'ensemble des quatre familles d'acides gras mono-insaturés en cis ou trans, à savoir : - la famille oléique en $n$-9, dont l'acide gras parent est l'acide oléique C18:1 cis;

- la famille palmitoléique en $n-7$ cis, dont l'acide gras parent est l'acide palmitoléique $\mathrm{C} 16: 1 \mathrm{n}-7$ cis ;

- la famille myristoléique en $n$-5, dont l'acide gras parent est l'acide myristoléique C14:1 n-5 cis;

- et la famille palmitoléique trans, dont l'acide gras parent est l'acide palmitoléique C16:1 n-7 trans.

En effet, comme nous le verrons, ces familles s'avèrent responsables d'effets biologiques majeurs.

\section{LA FAMILLE OLÉIQUE EN N-9 (INSATURATION DE TYPE CIS)}

\section{Classification de J. Mead}

Famille oléique

$\mathrm{C} 18: 0 \rightarrow \mathrm{C} 18: 1 \rightarrow \mathrm{C} 18: 2 \Rightarrow \mathrm{C} 20: 2 \rightarrow \mathrm{C} 20: 3 \ldots$ ? $n-9(\omega 9)$

$\Rightarrow$ Élongation

$\rightarrow$ Désaturation

L'acide oléique est le plus abondant des acides gras mono-insaturés à chaîne longue dans notre organisme. Sa formule chimique brute est $\mathrm{C}_{18} \mathrm{H}_{34} \mathrm{O}_{2}$ (ou $\mathrm{CH}_{3}\left(\mathrm{CH}_{2}\right)_{7} \mathrm{CH}=\mathrm{CH}\left(\mathrm{CH}_{2}\right)_{7} \mathrm{COOH}$ ). La particularité de l'acide oléique C18:1 n-9 repose sur l'importance de sa source alimentaire, au regard des synthèses endogènes possibles. Son caractère soi-disant neutre, autorisant par conséquent des apports sans limite de 10 à $20 \%$ de l'énergie totale, s'avère être une profonde erreur : sur le plan biologique, l'acide oléique est très contrôlé et forme la base de l'une des régulations les plus importantes en biochimie nutritionnelle. 


\section{Les sources exogènes d'acide oléique}

La source la plus traditionnelle d'acide oléique est l'huile d'olive, qui contient 72 à $73 \%$ d'acide oléique, suivie par le colza (60\%), l'arachide (46\%), le tournesol oléique ${ }^{36}$ (80 à $92 \%$ ) et l'huile d'argan (45\%), ainsi que l'huile de palme (39\%). Il existe bien entendu d'autres sources d'usage moins courant.

La matière grasse du lait apporte de 22 à $29 \%$ d'acide oléique, selon les saisons.

Ainsi, l'acide oléique exogène est-il l'acide gras le plus répandu dans la nature. On ne connaît qu'une seule situation où son apport, ou une cinétique de son métabolisme, se révèle sans doute insuffisant : la meibomite, une inflammation des glandes de Meibomius situées dans l'épaisseur de la paupière et en charge de fabriquer le meibum, la couche lipidique des larmes qui permet d'éviter leur trop rapide évaporation. Cette pathologie, qui se caractérise par un écoulement lacrymal et une atteinte chez les personnes très âgées de l'intégrité du bord de la paupière (décrit par Ntambi), peut être enrayée ou guérie par un apport d'acide oléique exogène, mais non par l'acide oléique endogène, ce qui suppose l'existence d'un circuit ciblé différentiel particulier.

\section{Les sources endogènes d'acide oléique}

L'acide oléique est non seulement l'acide gras le plus répandu dans la nature, mais c'est également celui dont la synthèse endogène a été la plus étudiée.

L'acide oléique provient de la désaturation en delta-9 de l'acide stéarique : C18:0 $\rightarrow \mathrm{C} 18: 1$ n-9.

36. La différence entre le tournesol oléique et le tournesol classique est la composition en acides gras de l'huile : comprise entre 15 et $25 \%$ pour le tournesol classique, la teneur en acide oléique atteint des valeurs de 80 à $92 \%$ pour les variétés oléiques. 
La désaturation en cis, située sur le neuvième carbone d'une chaîne qui en compte 18, est donc équidistante des deux extrémités de la molécule, ce qui en fait une désaturation pivot.

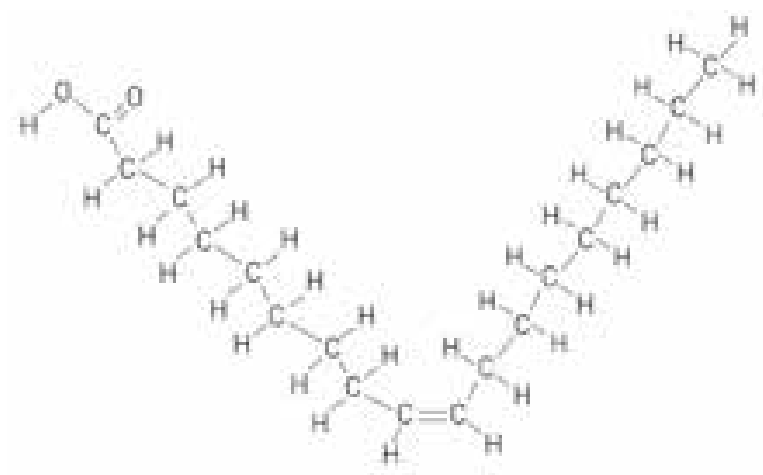

Figure 8 | Acide oléique.

Longtemps, la régulation de cette désaturation en delta-9, particulièrement sensible à l'insuline, a été l'objet de nombreux travaux, sans prendre en compte qu'elle succédait à l'élongation de l'acide palmitique C16:0 $\rightarrow$ C18:0, strictement dépendante de l'élongase ELOVL-6. Or, cette élongation est également insulinodépendante chez l'Homme. La synthèse endogène de l'acide oléique est donc doublement contrôlée.

\section{Voies métaboliques de la famille oléique}

Il existe au moins deux voies métaboliques de la famille oléique : une voie principale et une voie de suppléance.

\section{- La voie principale}

La voie métabolique principale de la famille des acides gras monoinsaturés en n-9 mène du C18:1 n-9 au C20:1 n-9 (acide gondoïque), au C22:1 n-9 (acide érucique) et enfin au C24:1 n-9 (acide nervonique) par une série d'élongations successives :

C18:1 $\omega 9$ (oléique) C20:1 $\omega 9$ (gondoïque) C22:1 $\omega 9$ (érucique) C24:1 109 (nervonique). 


\section{À propos de l'acide nervonique}

L'acide nervonique est connu depuis les années 1965-1970 comme étant le cinquième acide gras en poids dans le cerveau :

$-1^{\mathrm{er}}=$ acide oléique,

$-2^{\mathrm{e}}=$ acide arachidonique $(\omega 6)$,

$-3^{\mathrm{e}}=\mathrm{DHA}(\omega 3)$,

$-4^{\mathrm{e}}=$ acide adrénique $(\omega 6)$,

$-5^{\mathrm{e}}=$ acide nervonique $(\omega 9)$.

L'ignorance absolue de cette voie par nombre de spécialistes des lipides, en dehors des néonatologues et de certains neurologues, conduisit la communauté «scientifique » à l'effarante explosion d'un principe de précaution reposant uniquement sur les études de nutrition animale et dénonçant de ce fait « une toxicité de l'acide érucique et de l'acide nervonique » dans les années 1970-1975 (voir encadré page suivante)! Vingt ans plus tard, seule la scientifique canadienne Joyce Beare-Rogers fit acte de repentance et reconnut s'être trompée.

Mais il fallut encore attendre 20 ans pour qu'une étude sérieuse sur les effets des acides gras mono-insaturés à longue ou très longue chaîne soit publiée ${ }^{37}$ : "Nos résultats suggèrent une association inverse entre la teneur des globules rouges en C22:1 n-9, mais non en C20:1 n-9, en C18:l n-9, ou C24:1 n-9, et le risque de maladies coronariennes, après une correction de Bonferroni, dans l'analyse dite Physicians' Health Study. "

Ainsi, paradoxalement, la voie principale, menant exclusivement à des acides gras mono-insaturés à très longue chaîne (C24:1 n-9), a été reconnue bien plus tard que la voie de suppléance, en principe secondaire, de la famille oléique.

37. Matsumoto C., Matthan N. R., Lichtenstein A. H., Gaziano J. M., Djoussé L. (2013) Red blood cell MUFAs and risk of coronary artery disease in the Physicians' Health Study. Am. J. Clin. Nutr., 98 (3), 749-754. 


\section{À propos de l'acide érucique}

La méconnaissance de l'acide érucique entraîna quelques " délires ", comme par exemple la polémique à propos de la présence de cet acide gras dans le colza et les graines de moutarde. En effet, l'acide érucique avait été considéré comme toxique dans les années 1960 suite à des travaux menés sur l'animal. Il s'est ensuivi un énorme effort de recherche sur la sélection de variétés pauvres en acide érucique pour l'alimentation humaine (variétés de colza dites « double zéro », qui affichent une teneur en acide érucique inférieure à $2 \%$ de la fraction lipidique et, accessoirement, une teneur en glucosinolates inférieure à 20 micromoles par gramme). L'acide érucique ne restaura son image que le jour où on lui reconnut une efficacité réelle dans le traitement préventif de l'adrénoleucodystrophie, une pathologie qui se manifeste notamment par une démyélinisation progressive du système nerveux central ou une insuffisance surrénale.

\section{La voie de suppléance}

La voie de suppléance de synthèse endogène des acides gras polyinsaturés de la famille oléique, très bien décrite par Mead, conduit : - du C18:1 n-9 au C18:2 n-9 grâce à une désaturation en delta-6,

- puis du C18:2 n-9 au C20:2 n-9 grâce à une élongation due à l'ELOVL-5,

- et enfin du C20:2 n-9 au C20:3 n-9 grâce à une désaturation en delta-5.

Elle aboutit ainsi au C20:3 n-9 delta-5 ou triène de Mead, capable de suppléer dans certains cas à des fonctions de l'acide arachidonique (C20:4 n-6, delta-5). Ce mécanisme de suppléance permet à l'organisme, dans un premier temps, de parer, plus ou moins, à un apport insuffisant en acide linoléique (acide gras parent de l'acide arachidonique), par exemple pour des rôles uniquement structurels. Mais ce remplacement demeure partiel et imparfait : si le triène de Mead peut contribuer à une fluidité de la membrane quasi identique à celle conférée par l'acide arachidonique, il ne peut en revanche 
remplir le rôle de précurseur de prostaglandines ${ }^{38}$, également rempli par l'acide arachidonique.

\section{Voie de suppléance ou voie à fonctionnalité propre ? Les enseignements des travaux de C. L. Curtis}

Dans les années 2000, C. L. Curtis travailla sur un modèle animal d'arthrite dégénérative. Le problème observé : les chondrocytes (cellules composant le cartilage) et les membranes synoviales articulaires, soumises à un stress mécanique fréquent, se comportent comme des «Resident ProDrug » (réserves internes de médicaments), prêtes à s'enflammer au moindre traumatisme. En effet, l'acide arachidonique assure la fluidité articulaire mais, revers de la médaille, intervient également dans l'inflammation. D'où l'idée de le « remplacer » par le triène de Mead (C20:3n-9) qui pourrait ainsi assurer « une fluidité membranaire équivalente » à celle offerte par l'acide arachidonique, mais avec un risque moindre de « poussée inflammatoire ».

Ces travaux constituent peut-être un élément de réponse à une question longtemps posée : pourquoi, même avec des régimes très riches en acide linoléique, le triène de Mead ne disparaissait jamais complètement ? En effet, le rapport C20:3 n-9/C20:4 n-6 (triène de Mead/acide arachidonique), témoin d'une insuffisance d'apport en acide linoléique, peut varier de 0,04 à 10 , ces valeurs extrêmes signant en général la proximité du décès. Les travaux de Curtis permettent d'envisager que le C20:3 $n-9$ pourrait jouer un rôle propre dans certaines membranes, à de très petites quantités. Et ce n'est que lors d'une carence en acide linoléique, associée souvent à un régime trop glucidique (hyperinsulinémie), que sa synthèse explose au-delà du taux basal. Ainsi, cette seconde voie endogène ne serait pas seulement une voie de suppléance, mais une seconde voie à fonctionnalité propre, capable d'exploser pour tenter de résoudre les problèmes posés par une carence en acide linoléique.

38. Médiateur lipidique qui agit entre autres sur les plaquettes, l'endothélium, l'utérus et les mastocytes. 


\section{Intérêt de l'acide oléique et de ses dérivés en nutrition}

\section{humaine "vraie "}

Ces vingt dernières années, deux courants contradictoires se sont affrontés :

- un courant défendu par Andrea Bonanome et le Pr Scott Grundy dans un premier temps ${ }^{39}$ : selon eux, face aux problèmes cardiovasculaires, l'acide oléique est parfaitement neutre et peut donc constituer une variable d'adaptation, jusqu'à être consommé à des niveaux très élevés atteignant 20 à $25 \%$ de l'apport énergétique, soit 90 à 100 g/jour. Cinq ans plus tard, le Pr Scott Grundy reconnaissait partiellement son erreur et recommandait que l'acide oléique ne représente pas plus de 16,5\% de l'apport énergétique total, dans l'attente d'études plus approfondies ;

- un autre courant s'appuyant sur les travaux de D. H. Blankenhorn de l'Atherosclerosis Research Institute (Los Angeles, ÉtatsUnis) qui, dès 1990, montrait, directement chez l'Homme grâce à une série de coronarographies, que le risque de nouvelles lésions d'athérosclérose évolue selon une courbe en $U$ en fonction de la consommation d'acide oléique : le risque de lésions est réduit de $70 \%$ (risque relatif de 0,3) par rapport aux sujets témoins quand l'acide oléique représente de 9,9 à 12,3\% de l'énergie totale, alors qu'il est multiplié presque par 5 (risque relatif de 4,9) lorsque l'acide oléique représente plus de 12,3\% de l'énergie totale, et plus particulièrement lorsqu'il dépasse $16 \%$ de l'énergie totale (surmortalité métabolique) ${ }^{40}$.

L'explication de cet effet pathologique de l'acide oléique lorsqu'il dépasse un certain taux fut apportée, au terme de très longues études, par Lawrence L. Rudell et son équipe (faculté de médecine de

39. Bonanome A., Grundy S. M. (1988) Effect of dietary stearic acid on plasma cholesterol and lipoprotein levels. N. Engl. J. Med., 318 (19), 1244-8.

40. Blankenhorn D. H., Johnson R. L., Mack W. J., el Zein H. A., Vailas L. I. (1990) The influence of diet on the appearance of new lesions in human coronary arteries. JAMA, 263 (12), 1646-1652. 
Winston-Salem, Caroline du Nord, États-Unis ${ }^{41}$ : "L'enrichissement du centre de la particule LDL en oléate de cholestérol accroît les liaisons avec les protéines-glycans de la paroi artérielle et favorise l'artériosclérose. »

Les travaux de D. H. Blankenhorn, ainsi que ceux de Chisa Matsumoto et Luc Djoussé cités plus haut, incitent à la prudence quant à des régimes alimentaires apportant un taux d'acide oléique supérieur à $12,5 \%$ de l'énergie totale, et particulièrement lorsque ce taux dépasse $16 \%$ de l'énergie totale. Il est probable qu'à de telles valeurs, la synthèse d'acide érucique, seul acide gras de cette série qui soit protecteur vis-à-vis du risque coronarien, soit bloquée.

\section{Syndrome dysglycémique du troisième âge}

Une expérience récente, à savoir l'étude française dite Alphalinolénage (de 2009 à 2011, 188 patients hospitalisés au département gériatrique de l'hôpital francilien Émile-Roux, France) que nous avons déjà présentée, a permis de constater que $63 \%$ des hommes et $46 \%$ des femmes âgés de 85 ans souffraient d'un syndrome dysglycémique, favorisé par un apport lipidique insuffisant ( $49 \mathrm{~g} / \mathrm{jour}$ ), compensé par un apport glucidique ${ }^{42}$. Cette situation déclenche une lipogenèse de novo : l'acide stéarique C18:0 augmente de $18 \%$, l'acide palmitoléique C16:1 $n-7$ bondit de $+37 \%$ et l'acide oléique C18:1 $n-9$ gagne 7,3\%. Cette lipogenèse compensatoire est réalisée à partir de glucides. Mais, à cet âge avancé, l'activité de l'élongase ELOVL-6, responsable de l'élongation de l'acide palmitique C16:0 en acide stéarique C18:0, est réduite. Aussi, afin de contourner l'obstacle de l'ELOVL-6, l'acide palmitoléique prend le relais.

41. Melchior J. T., Sawyer J. K., Kelley K. L., Shah R., Wilson M. D., Hantgan R. R., Rudel L. L. (2013) LDL particle core enrichment in cholesteryl oleate increases proteoglycan binding and promotes atherosclerosis. J. Lipid Res., 54 (9), 2495-2503.

42. Henry O., Combe N., Vaysse C., Lopez C., Driss F., Fonseca I., Simon N., Le Guillou C., Masselin-Silvin S., David J.-P., Mendy F. (2015) Metabolic disorders and blood fatty acids status in hospitalized very old patients: Part I of the Alpha-linolenage study. OCL, 22 (3), D305. 


\section{LA FAMILLE PALMITOLÉIQUE N-7 (INSATURATION DE TYPE CIS)}

\section{Classification de J. Mead}

Famille palmitoléique

$\mathrm{C} 16: 0 \rightarrow \mathrm{C} 16: 1 \rightarrow \mathrm{C} 16: 2 \Rightarrow \mathrm{C} 18: 2 \rightarrow \mathrm{C} 18: 3 \Rightarrow \mathrm{C} 20: 3 \rightarrow \mathrm{C} 20: 4 n-7$

$\Rightarrow$ Élongation

$\rightarrow$ Désaturation

L'acide palmitoléique, de formule chimique brute $\mathrm{C}_{16} \mathrm{H}_{30} \mathrm{O}_{2}$ (ou $\left.\mathrm{CH}_{3}\left(\mathrm{CH}_{2}\right)_{5} \mathrm{CH}=\mathrm{CH}\left(\mathrm{CH}_{2}\right)_{7} \mathrm{COOH}\right)$, est également appelé acide cispalmitoléique. Bien que décrite dès 1955-1960 par J. Mead, en même temps que la famille oléique, la famille palmitoléique en $n-7$ restera pendant près de 40 ans quasi ignorée, en dehors des néonatalogues et des spécialistes des mucoviscidoses. L'acide palmitoléique est donc l'acide gras parent de la famille palmitoléique en $n-7$ cis.

\section{Source exogène d'acide palmitoléique}

L'acide palmitoléique est peu répandu. Les deux sources végétales les plus conséquentes sont l'huile de noix de macadamia (26\% des acides gras) et l'huile d'avocat $(7,3 \%)$. On en trouve également des traces dans l'huile de palme et de colza.

La seule source exogène notable est en réalité la matière grasse lactique, dans laquelle l'acide palmitoléique représente 2,6\% des acides gras.

\section{Source endogène d'acide palmitoléique}

À l'inverse de l'acide oléique, la quasi-totalité de l'acide palmitoléique est d'origine endogène. Il provient de la désaturation en delta-9 de l'acide palmitique C16:0, sous l'action de la même désaturase que celle désaturant l'acide stéarique C18:0 en acide oléique C18:1 n-6. Cette désaturation s'avère également insulinodépendante. Il existe une relation entre les concentrations en acides palmitoléique et palmitique qui ne semble pas être seulement le fruit du hasard : 
la proportionnalité d'un facteur 10 entre les deux acides gras laisse entrevoir une régulation parallèle.

\section{Voies métaboliques de la famille palmitoléique}

Deux voies métaboliques de la famille palmitoléique coexistent : une voie principale et une voie secondaire.

\section{- La voie principale}

La voie principale est celle des acides gras polyinsaturés endogènes en n-7, qui fut décrite par J. Mead en 1955-1960. Elle permet le passage :

- du C16:1 n-7 au C16:2 n-7 par une désaturation en delta-6 ;

- puis du C16:2 n-7 par une élongation (grâce à ELOVL-5) pour aboutir au C18:2 n-7, avant une désaturation en delta- 5 qui permet d'aboutir au C18:3 (n-7) ;

- une élongation (toujours grâce à l'ELOVL-5) aboutit au C20:3 $n-7$;

- enfin, c'est sans doute une seconde désaturation en delta-6 qui permet d'aboutir au C20:4 n-7, comme pour la série (n-3).

La particularité de cette voie principale est d'échapper complètement à la régulation de l'élongase ELOVL-6, qu'elle contourne.

\section{Identification du C22:4 $n-7$}

En chromatographie en phase gazeuse (CPG), technique qui permet de séparer des molécules d'un mélange, le C22:4 n-7 migre avec l'acide arachidonique, dont il a été longtemps difficile de le séparer sur le plan analytique. Par la suite, notre expérience montra que son taux s'avère particulièrement sensible aux carences en oméga-3, combinées à celles en oméga-6.

\section{La voie secondaire}

La voie secondaire débute par une élongation permettant la synthèse de l'acide cis-vaccénique C18:1 n-7 à partir de l'acide 
palmitoléique C16:1 n-7 cis. Comme dans la voie principale, l'élongase ELOVL-6 est contournée. En revanche, l'acide cis-vaccénique s'est révélé toxique (alors que sa version trans, le C18:1 n-7 trans, a un effet bénéfique comme nous le verrons plus loin !) : le travail de J. H. Wu et R. N. Lemaitre (Département de médecine cardiovasculaire, Brigham and Women's Hospital and Harvard Medical School, Boston, États-Unis) publié en 2011 dans The American Journal of Clinical Nutrition ${ }^{43}$, a montré qu'il était associé à un risque élevé de crise cardiaque chez l'Homme (risque plus de sept fois plus élevé après un ajustement multivariable). Nous y reviendrons dans notre chapitre dédié aux acides gras trans.

\section{Intérêt du palmitoléique en nutrition humaine}

Pendant 40 ans, l'acide palmitoléique n'a suscité l'intérêt que des seuls néonatologues et spécialistes de la mucoviscidose. Chez le foetus, il avait été observé qu'à la $32^{\mathrm{e}}$ semaine, le C16:1 n-7 représente 6,5\% des acides gras, contre seulement 3,1\% dans le même temps dans le sang maternel. Chez sept adolescents souffrant de mucoviscidose, la concentration sanguine en acide palmitoléique $\mathrm{C} 16: 1 n-7$ représentait $6,3 \%$ des acides gras totaux.

Ce n'est qu'en 2013 qu'une explication fut enfin proposée par K. M. Macrae et al. (Département de signalisation cellulaire et d'immunologie du Sir James Black Centre, université de Dundee, Royaume-Uni) dans un article publié dans le Journal of Lipid Research ${ }^{44}$ : "Une surcharge lipidique au niveau du muscle squelettique déclenche un syndrome inflammatoire qui est limité et

43. Wu J. H., Lemaitre R. N., Imamura F., King I. B., Song X., Spiegelman D., Siscovick D. S., Mozaffarian D. (2011) Fatty acids in the de novo lipogenesis pathway and risk of coronary heart disease: The Cardiovascular Health Study. Am. J. Clin. Nutr., 94 (2), 431-438.

44. Macrae K., Stretton C., Lipina C., Blachnio-Zabielska A., Baranowski M., Gorski J., Marley A., Hundal H. S. (2013) Defining the role of DAG, mitochondrial function, and lipid deposition in palmitate-induced proinflammatory signaling and its counter-modulation by palmitoleate. J. Lipid Res., 54 (9), 2366-2378. 
contre-régulé par l'acide palmitoléique. » Dans les deux cas (fœus et malade atteint de mucoviscidose), on comprend l'intérêt de cette contre-régulation : l'enfant souffrant de muscoviscidose lutte pour accroître la fluidité du mucus qui obstrue ses bronches en augmentant la concentration d'acide palmitoléique (plus fluide que l'acide palmitique) ; le foetus a des besoins énormes en acide palmitique et accroît en parallèle les concentrations d'acide palmitoléique pour se protéger.

En parallèle était progressivement décrit le rôle de l'acide palmitoléique dans la lipogenèse de novo à partir de glucides, contournant la régulation de l'élongase ELOVL-6, mais protégeant aussi le muscle squelettique d'une surcharge lipidique et véritable marqueur d'une lipogenèse hépatique.

Mais c'est un troisième rôle, en l'occurrence la description de l'acide palmitoléique comme une nouvelle "lipokine », hormone lipidique reliant le tissu adipeux au métabolisme systémique, par l'équipe américaine de la Harvard School of Public Health de Boston comprenant H. K. Cao et G. S. Hotamisligil ${ }^{45}$, qui a fait exploser l'intérêt porté à l'acide palmitoléique. Ainsi, le tissu adipeux pourrait avoir un impact direct sur l'homéostasie métabolique de l'organisme par le biais de sa composition et de sa sécrétion d'acides gras. La suite des travaux n'a pas tout à fait répondu aux attentes. Si le terme de lipokine reste contesté, un rôle très important de l'acide palmitoléique est reconnu. Et comme nous le verrons plus loin, la dissociation entre les effets physiologiques opposés des formes cis et trans de l'acide palmitoléique (et de l'acide vaccénique $n-7$ ) a ouvert un très important chapitre.

45. Cao H., Gerhold K., Mayers J. R., Wiest M. M., Watkins S. M., Hotamisligil G. S. (2008) Identification of a lipokine, a lipid hormone linking adipose tissue to systemic metabolism. Cell, 134 (6), 933-944. 


\section{LA FAMILLE MYRISTOLÉIQUE $N$-5 (INSATURATION DE TYPE CIS)}

L'acide myristoléique, ou acide 9-tétradécénoïque C14:1 n-5 cis, est un acide gras mono-insaturé de formule chimique $\mathrm{C}_{14} \mathrm{H}_{26} \mathrm{O}_{2}$. Il s'agit de la troisième famille créée par la désaturase en delta-9 ${ }^{46}$. D'ailleurs, c'est en étudiant les effets et la régulation de cette désaturase que l'on a découvert l'existence de cette famille en $n-5$ (désaturation située sur le cinquième carbone à compter de l'extrémité méthyle).

\section{Source exogène d'acide myristoléique}

L'acide myristoléique est pratiquement absent des sources végétales habituelles. Seule exception : l'huile des graines de myristicacées, une famille de plantes qui comprend notamment le muscadier, certaines de ces huiles étant constituées de près de $30 \%$ d'acide myristoléique.

En revanche, l'acide myristoléique est présent dans la matière lactique, où il compte pour 1,4 à 2,6 \% des acides gras totaux, avec une curieuse relation entre sa concentration et celle de l'acide myristique (l'acide myristoléique représente en général de l'ordre de $10 \%$ du taux d'acide myristique).

\section{Le noyau primordial de la matière grasse laitière}

L'acide myristoléique fait partie de cette fraction très particulière de la matière grasse lactique, décrite chez le lait des Mammifères dès 1968 comme le noyau primordial, ou PT de novo, génétiquement déterminé, ne dépendant pas de l'apport alimentaire. Cet ensemble comprend : - l'acide myristique C14:0, essentiellement en sn-2 donc protégé au centre des triglycérides, qui constitue en général $52 \%$ du noyau primordial ; - l'acide myristoléique C14:1 n-5 cis ${ }^{47}$;

- et un ensemble d'acides gras centrés autour d'une double liaison $n-7$ trans (acides trans-palmitoléique, trans-vaccénique, ruménique et C18:2 $n-5$ cis, $n-7$ trans). 


\section{Voies métaboliques de la famille myristoléique}

Les voies métaboliques de la famille myristoléique commencent seulement à être décrites. Une voie mène de l'acide myristoléique (C14:1 n-5 delta-9) au C16:1 n-5 delta-11 grâce à l'élongase ELOVL 1. Cette voie fait partie de la lipidogenèse de novo et aboutit à une liaison en delta-11 dangereuse, comme celle de l'acide vaccénique cis. Mais du fait de faibles valeurs en acide myristoléique, cette voie est peu utilisée.

Une seconde voie est privilégiée : celle de la synthèse par bêta-oxydation de l'acide oléique C18:1 n-9 en C16:1 n-9 (delta-11), malgré le caractère étonnant de cette bêta-oxydation dans un contexte de lipogenèse de novo.

\section{Intérêt de l'acide myristoléique en nutrition humaine}

Si l'intérêt de l'acide myristoléique commence aujourd'hui à être reconnu, la reconnaissance de son rôle n'en est qu'à ses balbutiements. Ainsi, la fonction régulatrice de cet acide gras, en complément de celle de l'acide myristique, se dessine progressivement. E. Montastier ${ }^{48}$ (Institut des maladies métaboliques et cardiovasculaires, Inserm UMR1048, Toulouse) vient de montrer, grâce à l'analyse de ses variations chez des femmes obèses soumises à des restrictions caloriques et lipidiques, le rôle inattendu de l'acide myristoléique C14:1 n-5 delta-9 cis dans cette régulation (voir encadré).

48. Douzièmes Journées francophones de nutrition, JFN 2014, Centre des congrès Le Square, Bruxelles, 10-12 décembre 2014. Publié dans : Montastier E., VillaVialaneix N., Caspar-Bauguil S., Gonzalez I., Saris W. H., Langin D., Kunesova M., Viguerie N. P204 : Omiques du tissu adipeux pour l'analyse des variations pondérales pendant et après une restriction calorique : une analyse de réseau pour l'intégration de données cliniques, du transcriptome et du lipidome. Nutrition Clinique et Métabolisme, 28, S175-176. 


\section{Les travaux de E. Montastier présentés lors des $12^{\text {es }}$ journées francophones de nutrition (2014)}

Le but du travail de cette équipe était d'étudier les relations entre des variables biocliniques d'individus obèses, l'expression des gènes et le contenu en acides gras du tissu adipeux. Et ce, à chaque étape d'un programme nutritionnel et en relation avec les variations pondérales.

Pour ce faire, l'analyse du contenu en acides gras du tissu adipeux sous-cutané abdominal et des mesures d'expression de 221 gènes ont été réalisées chez 135 femmes obèses de la cohorte européenne DiOGenes, avant le régime, après une phase de restriction calorique de 8 semaines et après 6 mois de suivi pondéral ad libitum. Les individus ont été séparés a posteriori en trois groupes selon les variations de poids après la restriction.

Après la phase de restriction, une relation positive forte entre l'acide myristoléique et des gènes codant pour les principales enzymes de la lipogenèse de novo a été observée. À la fin de la phase de suivi, dans le groupe de femmes qui ont continué à perdre du poids, cette relation entre acide myristoléique et gènes de la lipogenèse de novo a été également retrouvée.

D'où la conclusion de l'équipe : "Cette approche originale de biologie des systèmes apporte un regard nouveau sur la réponse du tissu adipeux au contrôle pondéral, en soulignant le rôle central et inattendu de l'acide myristoléique, qui pourrait être un déterminant de l'impact métabolique bénéfique de la perte de poids. »

\section{LA FAMILLE PALMITOLÉIQUE TRANS N-7}

Cette famille du palmitoléique C:16 n-7 trans, en pleine évaluation, a pour principale caractéristique une double liaison en trans en position $n-7$ (septième carbone à partir du groupement méthyle). 
À partir de l'acide gras parent C16:1 n-7 trans, quatre extensions s'avèrent possibles :

- soit vers l'extrémité carboxyle :

- une élongation donnant naissance au C18:1 n-7 trans (ou, pour les chimistes, une liaison trans située en delta-11, à savoir sur le onzième carbone - sur les 18 que comptent la chaîne carbonée - si l'on compte à partir du groupement carboxyle), ou acide vaccénique en $n$-7 trans (ou delta-11 trans);

- puis, après une désaturation en delta-9 cis, le C18:1 n-7 trans devient C18:2 n-7 trans, delta-9 cis, ou acide ruménique (porteur de deux insaturations : une insaturation trans en delta-11 issue de la molécule mère et une autre nouvellement formée en cis en delta-9) ;

- soit vers l'extrémité méthyle :

- une élongation et une désaturation menant au C18:2 n-5 cis, n-7 trans, qui s'avère être le deuxième acide gras trans, après l'acide ruménique, du noyau primordial de la matière grasse laitière ${ }^{49}$;

- et, après désaturation en delta- 9 du C18:2 n-5 cis, n-7 trans, l'obtention de l'acide gras C18:3 n-5 cis, connu sous le nom d'acide punicique ou pomégranique (majoritaire dans les pépins de la grenade) dans le monde végétal, porteur de trois insaturations : une insaturation cis en delta-9 nouvellement formée (ou n-9 pour les biologistes), une insaturation en trans en delta-11 (ou $n-7$ pour les biologistes) et une insaturation en cis en delta-13 (ou insaturation $n-5$ cis pour les biologistes).

La famille palmitoléique trans remet profondément en cause la question des acides gras trans physiologiques que l'on a opposés aux acides gras trans d'origine industrielle, touchant essentiellement la double liaison $n$ - 9 trans.

Elle attire également l'attention sur un acide gras particulier, le C16:1 n-7 trans ou acide palmitoléique trans, très grande originalité

49. L'origine première de la double liaison $n-5$ cis ne peut être que le monde végétal (indispensabilité $n-5$ cis ?). 
de la matière grasse lactique, au rôle physiologique reconnu comme étant plus important chaque jour.

\section{La désaturase en delta-13 attaque l'extrémité méthyle des acides gras... et un dogme ?}

Très récemment, Vincent Rioux (Laboratoire de biochimie-nutrition humaine, Agrocampus Ouest, INRA USC2012, Rennes, France) a décrit chez les rongeurs, dans un travail publié au sein de la revue Journal of Lipid Research ${ }^{50}$, une delta-13-désaturase capable de désaturer en delta-13 l'acide trans-vaccénique C18:1 (déjà porteur d'une insaturation dite en $n-7$ trans par les biologistes, ou delta-11 trans dans la nomenclature des chimistes), donnant donc naissance à une double liaison située en $n-5$ cis (le treizième carbone à partir du groupement carboxyle des chimistes correspond au cinquième carbone à partir du groupe méthyle des chimistes, sur cette chaîne de 18 atomes de carbone). La molécule obtenue, appelée acide linoléique conjugué trans 11, cis 13 (ou CLA, pour conjugated linoleic acid), est déjà décrite dans le noyau primordial de la matière grasse lactique. Or, on avait jusque-là toujours imaginé que, à l'inverse des plantes, le monde animal était caractérisé par l'absence de désaturases introduisant des doubles liaisons du côté du groupe méthyle (les insaturations en delta-5, 6 ou 9 se produisent du côté carboxyle). Cette désaturase en delta-13 apparaît donc, pour le moment, comme la seule désaturase s'attaquant à l'extrémité méthyle des acides gras caractérisée chez les animaux ( $n-5$, intermédiaire entre $n-3$, et $n-6)$.

\section{Intérêt de la famille de l'acide palmitoléique trans $n-7$ en nutrition humaine}

L'importance de la famille palmitoléique est soulevée pour la première fois dans un travail publié en 2010 sous le titre «Dietary 
Trans-Palmitoleic Acid and Diabetes ${ }^{51}$ : un effet bénéfique de l'acide palmitoléique ayant été suggéré, l'équipe a suivi 3736 hommes et femmes âgés de 65 ans ou plus, qui ont accepté de se soumettre à des entrevues et examens pendant de nombreuses années afin d'identifier les facteurs de risque de diverses maladies. Les chercheurs ont d'abord demandé aux participants de remplir un questionnaire sur les aliments qu'ils mangeaient habituellement et sur d'autres facteurs qui pourraient affecter leur santé. Trois ans plus tard, des échantillons de sang ont été prélevés pour mesurer les niveaux d'acides gras (y compris d'acide palmitoléique trans). Les participants ont ensuite été évalués chaque année pour déterminer d'éventuels problèmes de santé. Les résultats montrent que les participants ayant déclaré manger davantage de matières grasses issues des produits laitiers avaient des niveaux plus élevés d'acide palmitoléique trans dans leur sang trois ans plus tard. Au cours des années suivantes, les participants qui avaient des niveaux plus élevés d'acide palmitoléique trans dans le sang présentaient davantage d'indicateurs de bonne santé (tels que certains types de cholestérol) et étaient moins susceptibles de développer un diabète.

Ces premiers résultats furent confortés par les travaux de la Harvard School of Public Health (Boston, États-Unis) et plus précisément par leur publication de $2013^{52}$ dans l'American Journal of Clinical Nutrition. L'étude repose sur la cohorte dite MESA, acronyme de Multi-Ethnic Study of Atherosclerosis, dont elle a retenu 2281 participants ne présentant pas de diabète parmi les 2617 participants de l'étude initiale. Dans cette population, 205 cas de

by FADS3 in rodents. J. Lipid Res., 54 (12), 3438-3452.

51. (2010) Dietary trans-palmitoleic acid and diabetes. Ann. Intern. Med., 153 (12), I1-56.

52. Mozaffarian D., de Oliveira Otto M. C., Lemaitre R. N., Fretts A. M., Hotamisligil G., Tsai M. Y., Siscovick D. S., Nettleton J. A. (2013) Trans-Palmitoleic acid, other dairy fat biomarkers, and incident diabetes: the Multi-Ethnic Study of Atherosclerosis (MESA). Am. J. Clin. Nutr., 97 (4), 854-861. 
diabètes sont apparus entre l'inclusion (en 2000-2002) et la fin de l'étude (2005-2007).

Après un ajustement multivariable, l'acide palmitoléique trans est associé :

- à un taux de LDL plus élevé de $6,4 \%(\mathrm{p}=0,005)$ (lié à un excès d'acide stéarique dans la matière grasse lactique ?) ;

- à un taux plus faible de triglycérides de $19,1 \%(\mathrm{p}=0,002)$;

- à un taux d'insuline à jeun plus bas de $9 \%(\mathrm{p}=0,002)$;

- à une pression systolique plus faible de $2,4 \mathrm{mmHg}(\mathrm{p}=0,01)$.

Au terme de l'analyse prospective, l'acide palmitoléique trans apparaît comme indépendamment associé à une incidence plus faible du diabète $(\mathrm{p}=0,02)$. Selon les auteurs, le risque de diabète serait réduit de $48 \%$ dans le groupe ayant la plus forte concentration sanguine en acide palmitoléique (donc les forts consommateurs de produits laitiers), comparativement au groupe ayant la concentration la plus faible.

\section{Brevets}

Forts de leurs résultats, les auteurs de l'étude de 2013, à savoir D. Mozaffarian et G. Hotamisligil, et l'université de Harvard ont déposé un brevet sur le C16:1 n-7 trans pour le dosage de l'association d'acides gras de leur étude. Ce brevet est d'ailleurs antériorisé par un brevet que $\mathrm{H}$. Dabadie et moi-même avions précédemment déposé (0851269-27/02/08) sur un produit laitier obtenu par cristallisation fractionnée, associant une augmentation de $50 \%$ des acides myristique et myristoléique, de $50 \%$ de l'acide palmitoléique trans et une réduction de $50 \%$ de l'acide stéarique. Il est de plus également antériorisé et élargi par un brevet européen de F. Mendy et L. Roger (PCT/EP2009053470) sur l'acide myristique en sn- $+n-5$ cis, n-7 trans, $n-5$ cis, $n-7$ trans, $n-9$ cis. 
Plus récemment, un travail de Maria Pina Mollica (université de Naples, Italie), publié en 2014 dans le Journal of Lipid Research ${ }^{53}$, a confirmé le rôle hautement protecteur de l'acide ruménique C18:2, cis 9, trans 11 vis-à-vis de la stéatose hépatique. L'activation de la biogenèse et de la fonction mitochondriale est décryptée et attribuée à la fonction trans 11 .

Ainsi, l'intérêt de l'élongation à partir de la liaison n-7 trans (ou trans 11 pour les chimistes) vers la formation d'une double liaison en $n-5$ cis (ou cis delta-13) se confirme. Cette dernière peut également être suivie d'une désaturation en delta-9 cis, ce qui aboutit principalement à un acide gras maintenant bien décrit : l'acide punicique ou pomégranique, C18:3 n-5 cis, porteur de trois insaturations (une insaturation cis en delta-9, une insaturation en trans en delta-11 et une insaturation en cis en delta-13), le fameux acide gras des pépins de grenade.

En réalité, avec cette double liaison $n-7$ trans et le motif combinant les liaisons $n-5$ cis, $n-7$ trans et $n-9$ cis, s'ouvre tout un domaine extrêmement novateur, couvrant à la fois les mondes végétal, animal et la biochimie humaine. Cet ensemble, qui va de l'acide oléique à l'acide palmitoléique $n-7$ trans et l'acide pucinique, devient l'un des sujets prometteurs de la biochimie et de la nutrition chez l'Homme.

53. Mollica M. P., Trinchese G., Cavalierea G., De Filippo C., Cocca E., Gaita M., Della Gatta A., Marano A., Mazzarella C., Bergamo P. (2014) c9,t11-Conjugated linoleic acid ameliorates steatosis by modulating mitochondrial uncoupling and Nrf2 pathway. J. Lipid Res., 55, 837-849. 


\section{Le rôle anti-inflammatoire de l'acide pucinique}

Un travail de chercheurs de l'Inserm (Inserm U773, université Paris Descartes, Faculté de médecine, France) publié en 2009 dans PLOS $O N E^{54}$, avait montré que l'acide pucinique exerçait un puissant effet anti-inflammatoire, à travers l'inhibition de la TNF-alpha pro-inflammatoire et de son action, sur la NADPH-oxydase et, par cascade, sur la myéloperoxydase, enzyme impliquée dans la réaction immunitaire qui exerce une forte activité antimicrobienne.

Des travaux japonais avaient montré pour leur part que, comparé à l'acide linoléique, l'acide pucinique diminuait fortement la sécrétion d'Apo B-100.

Plus récemment, Spilmont et Coxam ${ }^{55}$ ont mis en évidence que l'acide pucinique et ses dérivés diminuaient les phénomènes inflammatoires et de stress oxydatif sur un modèle animal d'ostéoporose post-ménopausique.

Ainsi, l'acide pucinique (également appelé acide pomégranique) apparaît comme la molécule la plus prometteuse dans la prévention ou le traitement du syndrome inflammatoire chronique latent ou de bas grade. Il est d'ailleurs intéressant de noter que des tentatives de sélection de colza riche en acide pucinique ont eu lieu.

54. Boussetta T., Raad H., Lettéron P., Gougerot-Pocidalo M. A., Marie J. C., Driss F., El-Benna J. (2009) Punicic acid a conjugated linolenic acid inhibits TNF alphainduced neutrophil hyperactivation and protects from experimental colon inflammation in rats. PLoS One, 4 (7), e6458.

55. Spilmont M., Léotoing L., Davicco M. J., Lebecque P., Miot-Noirault E., Pilet P., Rios L., Wittrant Y., Coxam V. (2015) Pomegranate Peel Extract Prevents Bone Loss in a Preclinical Model of Osteoporosis and Stimulates Osteoblastic Differentiation in Vitro. Nutrients, 7 (11), 9265-9284. 


\section{» À retenir}

L'acide oléique C18:1 n-9 (insaturation de type cis en position delta-9) est l'acide gras le plus répandu dans la nature et celui dont la synthèse endogène a été la plus étudiée. Deux voies de synthèse coexistent : une voie principale menant, d'élongation en élongation, à l'acide nervonique C24:1 n-9, et une voie de suppléance décrite depuis les années 1960 par J. Mead menant au triène de Mead ou C20:3 n-9, porteur de trois insaturations. Côté nutrition, il semble que des apports en acides oléiques supérieurs à $12,5 \%$ représentent un risque coronarien encore plus marqué pour un taux supérieur à $16 \%$ de l'apport énergétique total. L'acide palmitoléique C16:1 n-7 cis (insaturation en position delta-9), peu répandu dans le monde végétal, est consommé via les matières grasses du lait, mais aussi fabriqué par notre organisme à partir de l'acide palmitique C16:0, qui subit une désaturation en delta-9. L'acide palmitoléique $116: 1 \mathrm{n}-7$ est lui-même l'acide gras parent de toute une famille obtenue via deux voies : celle, principale, des acides gras polyinsaturés en $\mathrm{n}-7$ décrite par J. Mead et une voie secondaire qui, par l'action d'une élongase, mène à un acide toxique, l'acide (cis-)vaccénique $118: 1$ n-7 cis (insaturation de type cis en position delta-11). Alors que, comme nous le rappelons plus loin, l'acide (trans-)vaccénique a un effet bénéfique, après transformation en acide ruménique $\mathrm{C18:2} \mathrm{n}-7$ trans, $\mathrm{n}-9$ cis. L'acide palmitoléique semble limiter et contre-réguler le syndrome inflammatoire. Certains travaux ont même évoqué un rôle de "lipokine ", faisant de cet acide gras une hormone lipidique qui pourrait permettre au tissu adipeux d'influer sur le métabolisme systémique.

L'acide myristoléique C14:1 n-5 cis, absent des sources végétales habituelles, est présent dans la matière lactique, où il compte pour 1,4 à $2,6 \%$ des acides gras totaux, et fait partie de ce que l'on appelle le noyau primordial, cette fraction particulière du lait. La famille de cet acide gras découle de deux voies de synthèse que l'on commence seulement à décrire, sachant que la synthèse par bêta-oxydation de l'acide oléique C18:1 n-9 en C16:1 n-9 est privilégiée, malgré le caractère étonnant de cette bêtaoxydation dans un contexte de lipogenèse de novo. L'acide myristoléique semble jouer un rôle régulateur en complément de celui de l'acide myristique, que les équipes de chercheurs commencent seulement à entrevoir. 
Enfin, l'acide palmitoléique C16:1 n-7 trans (insaturation de type trans en position delta-9) est actuellement au centre de nombreux travaux de recherche, notamment en raison de cette double liaison de type trans en $\mathrm{n}-7$ qui, après élongation, va devenir une liaison trans en $\mathrm{n}-11$ dans un acide gras dérivé de la famille: l'acide (trans-)vaccénique. Trois autres acides gras sont dérivés de l'acide gras parent palmitoléique : l'acide ruminique C18:2 n-7 trans delta-9 cis, l'acide gras C18:2 n-5 cis, n-7 trans et l'acide punicique $\mathrm{C} 18: 3 \mathrm{n}-5$ cis, n-7 trans, $\mathrm{n}-9$ cis. Leurs rôles en nutrition humaine sont progressivement dévoilés : réduction de l'incidence du diabète par l'acide palmitoléique $616: 1 \mathrm{n}-7$ trans, protection de la stéatose attribuée à la fonction trans-11, rôle de l'acide pucinique dans le processus anti-inflammatoire, etc.

La double liaison de l'acide myristoléique (C14:1, n-5 cis, Delta-9 cis) s'avère de plus en plus indispensable, or non synthétisable, chez l'homme (en plus des doubles liaisons en $n-3$ et en $n-6$ ). Elle s'intercale entre ces deux doubles liaisons $n-6$ et $n$-3. Cet acide gras mono-instauré est le point de départ, avec également le C16:2, n-5 cis, n-7 trans d'origine laitière, de la synthèse de l'acide punicique (C18:3,n-5 cis, n-7 trans, $n-9$ cis), présent également dans l'huile de pépins de grenade. Non synthétisable en l'absence de la double liaison n-5 cis, malgré la possibilité de synthèse des doubles liaisons $n-7$ trans, et $n-9$ cis, il semble que l'absence d'acide punicique puisse faire naitre un acide gras très voisin, le C18:3, n-5 trans, n-7 cis, n-9 trans, sorte de miroir. Il est possible que la compréhension des rôles respectifs de ces deux acides gras puisse faire avancer nos connaissances dans la compréhension du syndrome inflammatoire latent ou de bas grade, extrêmement dangereux sur le long terme. 